PACS: $71.25 . \mathrm{Rk}, 81.60 \mathrm{Cp}$

\title{
Two-dimensional photonic crystals as perspective materials of modern nanoelectronics
}

\section{L.A. Karachevtseva}

V. Lashkaryov Institute of Semiconductor Physics, NAS of Ukraine, 41, prospect Nauky, 03028 Kyiv, Ukraine Phone: (8-044) 525-98-15. Fax: (8-044) 525-83-42

E-mail: lakar@isp.kiev.ua

\begin{abstract}
Photonic crystals are a dynamic direction of modern solid state physics. Today the intensive research (more than $80 \%$ ) is concentrated on two-dimensional photonic crystals which have functionality of three-dimensional photonic crystals and rather simple manufacturing techniques and is directed towards the development of active and passive elements of the future integrated nanophotonic circuits. Twodimensional photonic macroporous silicon structures are perspective for development of photonic waveguides, thermal receivers due to occurrence of the powerful absorption bands in the infrared spectrum range. Formation macroporous silicon structures with nanocoatings expands its functionalities as photodetectors and light emitters.
\end{abstract}

Keywords: two-dimensional photonic crystals, nanoelectronics, macroporous silicon.

Manuscript received 14.10.04; accepted for publication 16.12.04.

\section{Introduction}

Photonic crystals are periodic structures with the modulated dielectric constant. The spectrum of electromagnetic radiation is classified into zones forbidden for its propagation similar to electronic bands in semiconductors. The principal works with the idea of photonic bandgap formation were published in 1987 $[1,2]$. However, this problem of solid state physics has received the dynamic development during last ten years. The application spectrum of the photon localization effect is rather wide: filters, reflectors, and substrates for antennas in the microwave range; waveguides which correct the distribution of light in optical fibres; zerothreshold lasers and low-loss resonators; electromagnetic wave filters. The effect of the photonic bandgap formation opens the perspectives to manufacture photon analogs of the semiconductor devices.

Photonic crystals can be divided into one-, two- and three-dimensional structures (Fig. 1), depending on quantity of directions of photonic bandgap formation.

Wave equation for electromagnetic irradiation in periodical structures has the common features with the Schrödinger equation for electrons in crystals:

$\left[-\frac{h^{2}}{2 m^{*}} \nabla^{2}+V(x)\right] \Psi(x)=E \Psi(x)$.

The monochromatic electromagnetic wave is described by the equation:

$$
-\nabla^{2} \vec{E}+\nabla(\nabla \vec{E})-\frac{\omega^{2}}{c^{2}} \varepsilon(x) \vec{E}=\frac{\omega^{2}}{c^{2}} \varepsilon_{0} \vec{E},
$$

which directly follows from the Maxwell equations. Thus, the electron wavefunction $\Psi(x)$ is corresponded to a vector of the electric field $\vec{E}$ of an electromagnetic wave. The electrical field of electron $\vec{E}$ corresponds to the dielectric constant $\varepsilon_{0}$. The periodical potential of electron electrical field $V(x)$ corresponds to the periodical dielectric constant $\varepsilon(x)$. The solution of the wave equation (2) for electromagnetic radiation in periodical structures is connected with the fourth-order determinant taking into account both $E$ - and $H$-components of electromagnetic field. There are no solutions of the wave equation in the region of photonic bandgap (Fig. 2).

\section{Two-dimensional photonic crystals}

Today the basic researches are concentrated on twodimensional photonic crystals that have functionality of three-dimensional photonic crystals and rather simple manufacturing techniques. So, currently researches of three-dimensional photon crystals take $2 \%$, onedimensional $-15 \%$, and two-dimensional consists more than $80 \%$ of the total scientific works. The most effective methods of the two-dimensional photonic crystal formation are X-ray and electron beam lithography; dry, ionic-beam and electrochemical etching. Such methods allow us to form structures of the sizes of air rods from $10 \mathrm{~nm}$ up to $10 \mu \mathrm{m}$. Formation of 
a)

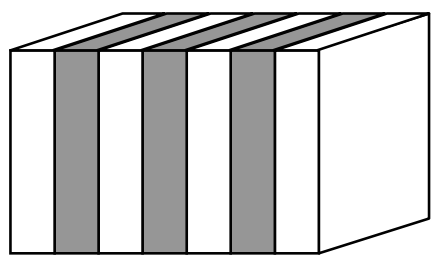

PBG

b)
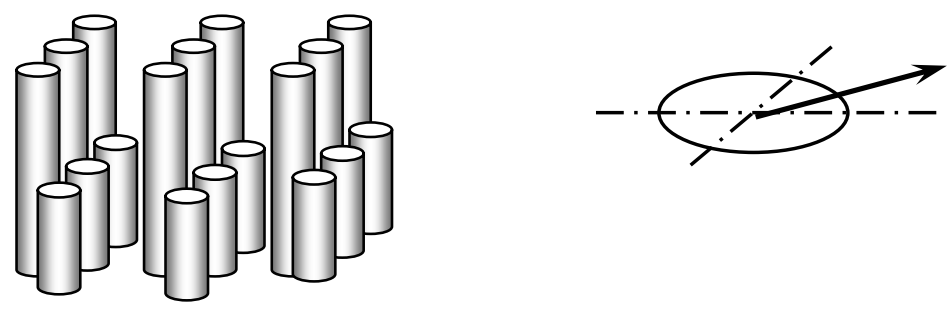

PBG

c)
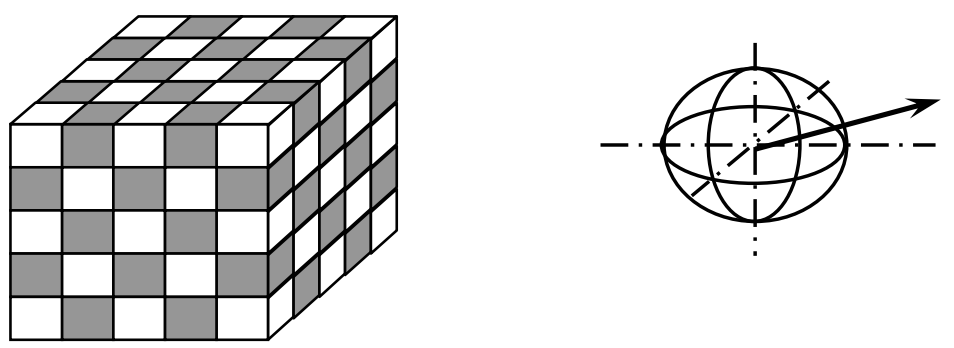

PBG

Fig. 1. One (a), two- (b) and three-dimensional (c) photonic crystals (PBG - a direction of the photonic forbidden zone).

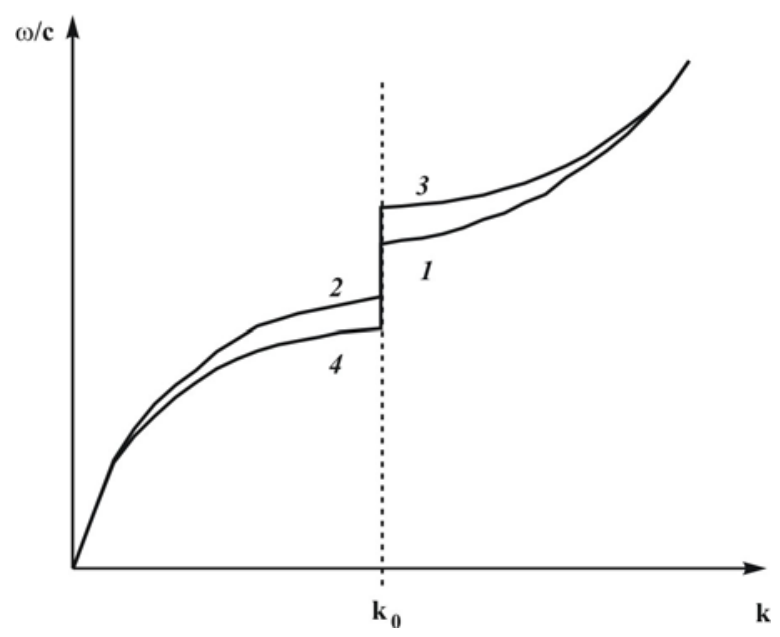

Fig. 2. Dependence of the longitudinal $(1,2)$ and crosssection $(3,4)$ frequency components of electromagnetic radiation on a wavevector.

structures without a line of rods permits to fabricate photonic waveguides. Electromagnetic waves are localized in the waveguide and penetrate due to the Bragg-type scattering. Formation of structures without single rod permits to fabricate cavities. Electromagnetic waves are enhanced due to localization in cavities.

Practical researches in the field of two-dimensional photonic crystals directed, mainly, towards the
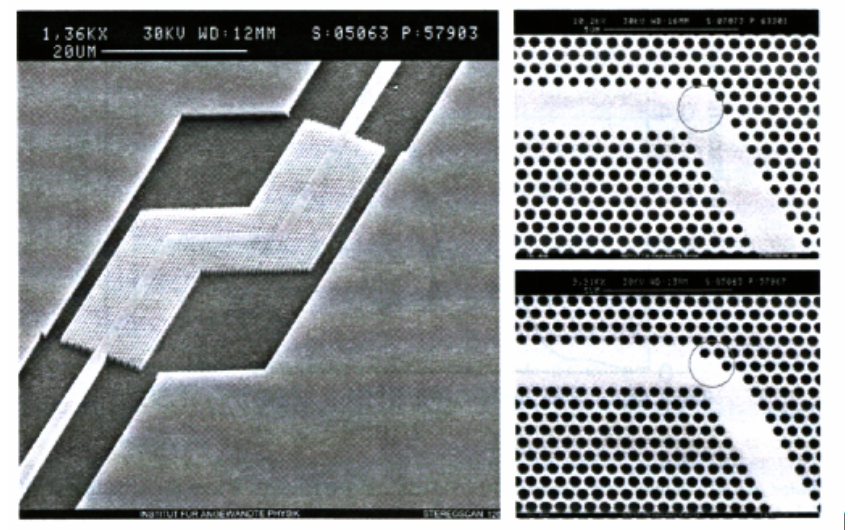

Fig. 3. Photonic crystal double bend realized in glass-like amorphous materials with the optimized (bottom right) bend design; diameter of rods is $374 \mathrm{~nm}$ [3].

development of the future integrated nanophotonic circuits. Active elements for transfering the energy are produced on a basis of illuminated structures $\mathrm{A}^{3} \mathrm{~B}^{5}$, passive (photonic waveguides, photonic fibres) - from silicon oxide, silicon [3]. Photonic waveguides have bandwidth of 50 terabits per second while the electronic transport provides some gigabits per second. Therefore, the important task of recent years is formation of 

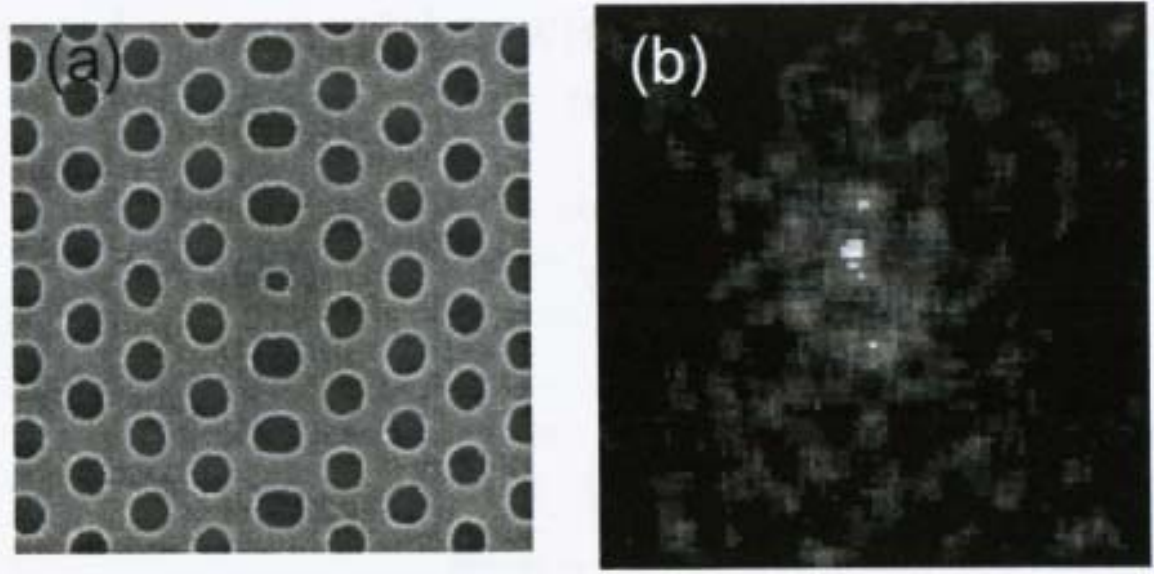

Fig. 4. Quantum well of photonic crystal nanolaser InGaAsP (a) and a near-field microscopy image of a superposition of orthogonal modes (b) [4].

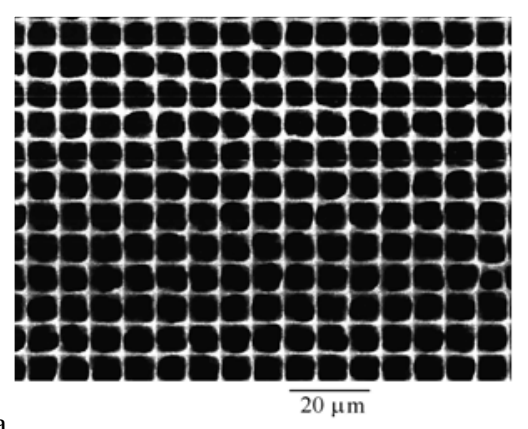

a

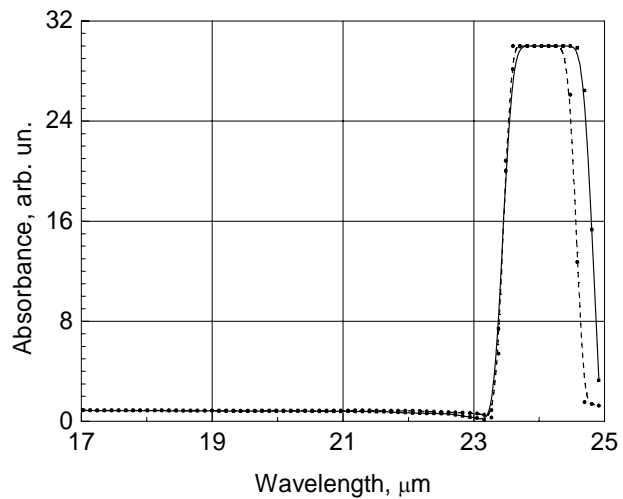

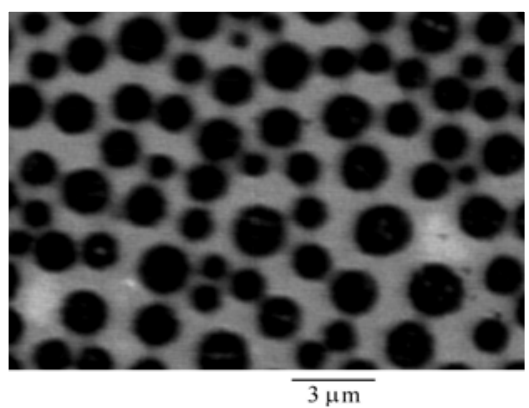

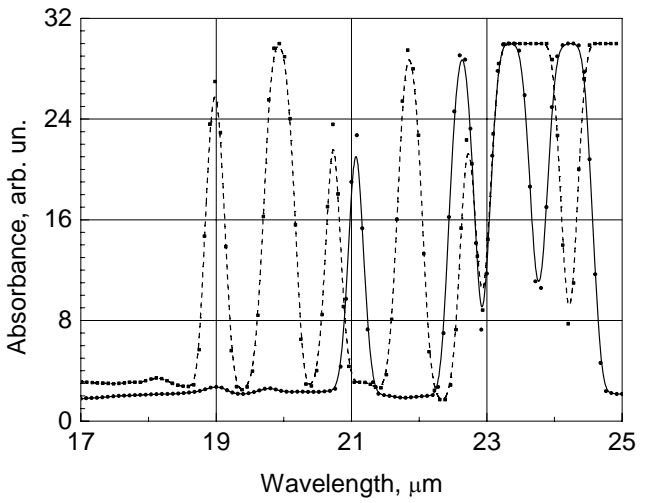

d

Fig. 5. Periodic (a) and arbitrary (b) structures of macroporous silicon. Optical absorption of electromagnetic waves propagated in periodic (c) and arbitrary (d) macroporous silicon.

waveguides on the basis of two-dimensional photonic crystals instead of copper interconnects in microcircuits due to higher bandwidth, lack of thermal heating. Through the next 5-7 years, such waveguides are supposed to be commercial devices. Modern researches in photonic waveguides are directed towards the maximal transfer of electromagnetic energy, in particular, towards design of points of turn (Fig. 3).
Development of two-dimensional photonic crystal nanolasers with quantum wells and quantum dots was demonstrated in [4]. Photonic crystal cavities with the quantum well active material are simple but powerful nanolasers to produce the intense laser output for signal processing (Fig. 4). Such structures have small surface recombination and losses by absorption, high speed, small thresholds. The quantum well is a structure, in 
which the central cylinder is absent (Fig. 4).

\section{Two-dimensional macroporous silicon structures}

One of the perspective materials for the development of two-dimensional photonic structures is macroporous silicon that can be obtained by the method of photoanodic etching (Figs 5a and b). It is connected with formation of structures of the necessary geometry, the high ratio of the cylindrical macropore depth to the diameter [5].

Optical absorption. The absolute photonic bandgap was measured at wavelengths between one and two optical periods $\lambda_{a}$ of macroporous silicon structure for a light direction parallel to macropores for planar technologies (Fig. 5c and d). Obtained results qualitatively correlate with theoretical calculations [6-8].

Absorption spectra of macroporous silicon with different pore diameters and concentrations have common features at $\lambda \leq \lambda_{a}$. There is an essential reduction in transmission of electromagnetic radiation with a shortening of the wavelength. Recalculated absorption coefficient is equaled to $300 \ldots 500 \mathrm{~cm}^{-1}$, as shown in Fig. 6. The transmission spectra contain steps. In the longwave range of the spectrum, the step frequency is proportional to the distance between the pores, and in the shortwave region it is proportional to the diameter of the pores. Transmission spectra of macroporous silicon as well as the formation of the steps can be explained by the model of directed and radiated optical modes [9]. Macroporous silicon is a short waveguide structure with a large contrast in dielectric constants of the pores and the silicon matrix. In the shortwave spectral region, the directed optical modes are formed in macropores. The longwave spectral region modes are apparently formed by Si matrix.

Thus, the macroporous silicon structures are perspective, in particular, to develop thermal detectors, photonic waveguides due to occurrence of the absorption bands in the infrared spectral range. Formation of the macroporous silicon structures with nanocoatings expands its functionalities.

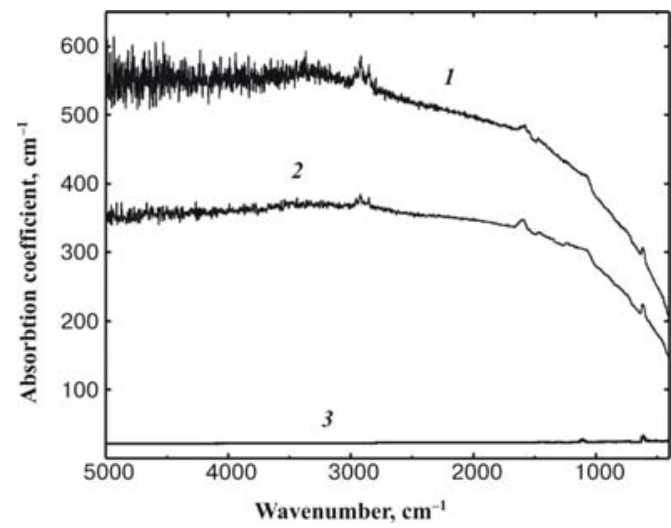

Fig. 6. Spectral distribution of the effective absorption coefficient: 1, 2 - macroporous silicon samples, 3 - silicon plate.

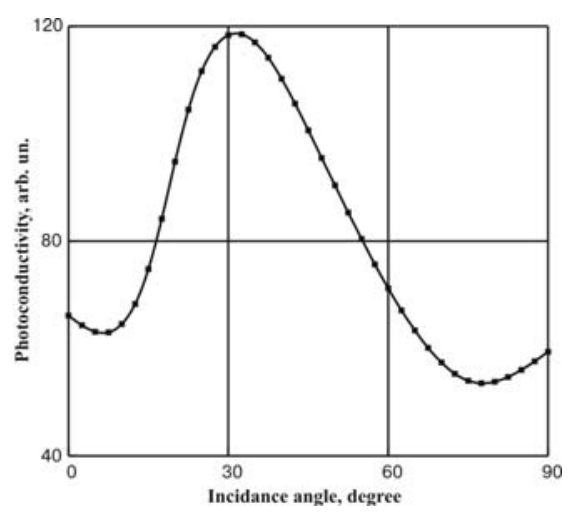

Fig. 7. Dependence of photoconductivity of the periodical macroporous silicon structure on the light incidence angle.
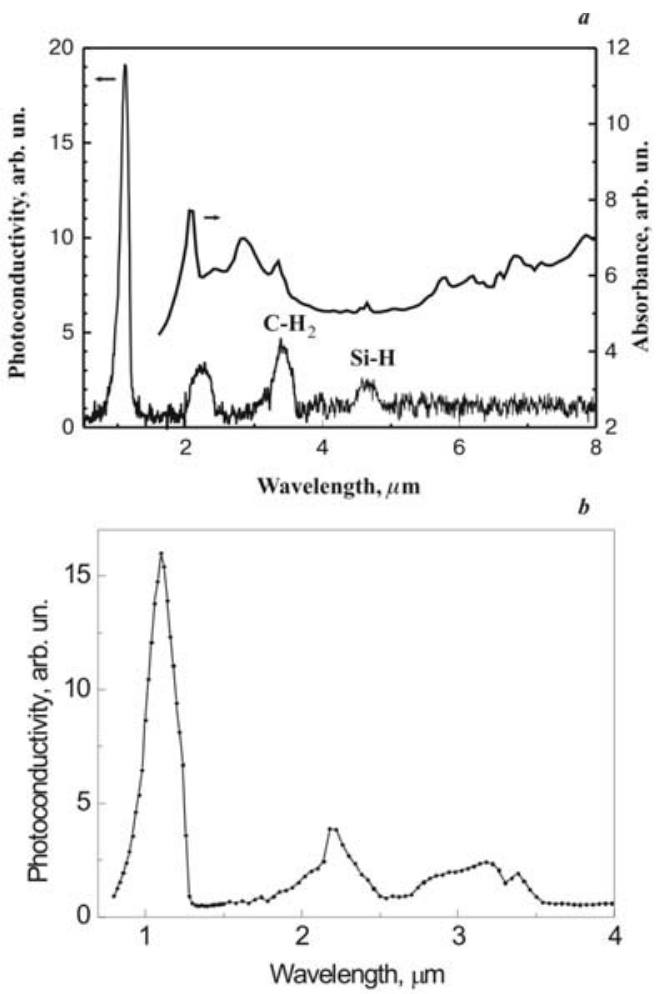

Fig. 8. Spectral dependences of photoconductivity and absorbance of macroporous silicon structures with nanocoatings: $a-\mathrm{SiO}_{2}, b-\mathrm{SiC}$.

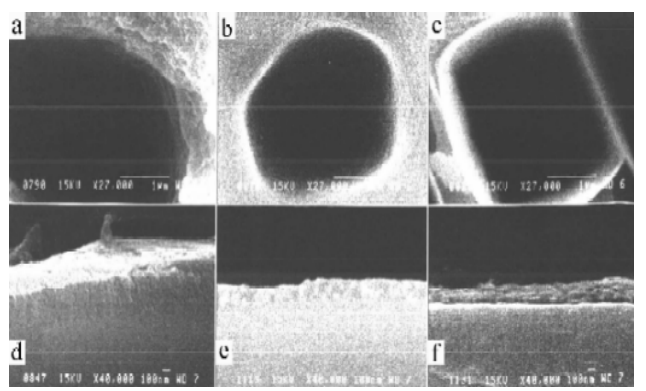

Fig. 9. Electron microscopic images of macropore outer surfaces $(a-c)$ and cross-sections of macropore walls $(d-f)$ at various current densities through macropores $j_{p}$ (in $\mathrm{mA} / \mathrm{cm}^{2}$ ): (a) and (d) - 40, (b) and (e) - 46, (c) and (f) $-54 \mathrm{~mA} / \mathrm{cm}^{2}$. 


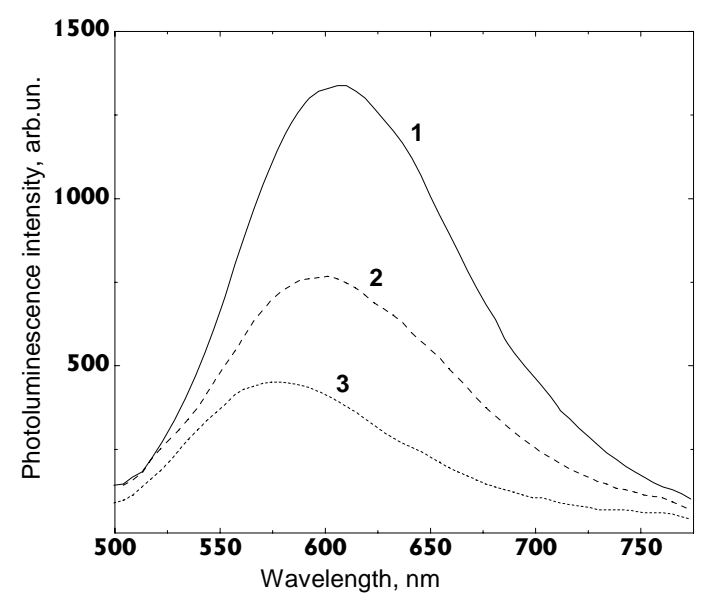

Fig. 10. Photoluminescence spectra of macroporous silicon structures formed by anodization at various current densities through macropores $j_{p}$ (in $\left.\mathrm{mA} / \mathrm{cm}^{2}\right)$ : (1) - 40, (2) - 46, and (3) 54 .

Photoconductivity. Photoelectrical properties of macroporous silicon structures are determined both by the periodic macropore distribution and the state of macropore surface. Elecrtroreflectance spectroscopy of macroporous silicon structures showed Franz - Keldysh oscillations, which is indicative of the development of intrinsic electric field caused by enrichment of the spacecharge region by major carriers. The intrinsic electric field of about $10^{6} \mathrm{~V} / \mathrm{cm}$ appears due to the positive charge built into an oxide layer on the walls of macropores [10].

Thus, the photoconductivity depends on a light incidence angle (Fig. 7). The dependence of photoconductivity on the angle of incidence of the electromagnetic radiation was observed to have a maximum at normal incidence of electromagnetic radiation, within the region of the angle of total internal reflection relative to the macropore walls and at a grazing angle of incidence respective to the surface of the structure. Angular dependences of photoconductivity, the excess of the light absorption over its reflection in the structures of macroporous silicon are determined by interaction of optical modes with oscillator fluctuations on the macropore surface and the surface polariton formation [11]. Photoconductivity maxima correlate with maxima of the intrinsic and impurity light absorption (surface, $\mathrm{C}-\mathrm{H}$ and $\mathrm{Si}-\mathrm{H}$ bonds in the enriched layer of macropore surface, Fig. 8a). Photoconductivity is determined by the conditions at the interface silicon silicon carbide also after $\mathrm{SiC}$ nanocoating formation on a macropore surface (Fig. 8b) using a low-temperature gastransport reactions. Amplification of photoconductivity of this structure reaches tenfold amplification in comparison to the monocrystalline silicon.
Photoluminescence. Macroporous silicon structures contain a layer of microporous silicon of 100 to $700 \mathrm{~nm}$ depth on macropore walls depending on a regime of the electrochemical process (Fig. 9). Photoluminescence of complex micropore-macropore silicon structures was investigated. The intensity of orange photoluminescence peaking near $600 \mathrm{~nm}$ (Fig. 10) decreased for higher current densities through the macropores [12]. Relaxation curves of a photoluminescence consist of several components as well as in flat microporous layers. Peaks of light absorption in such structures are intensive due to high porosity and the large common area of the surface of microporous layers. Perspectives for applying these complex structures of macroporous silicon with nanocrystals of microporous layers are, first of all, in the geometrical factor bound to the large common area of the illuminated surface and opportunity of the radiation amplification by these periodic structures at commensurable values of the light wavelength and structure periods.

\section{Conclusions}

Photonic crystals are a dynamic direction of solid state physics. The basic practical development stems from the possibility to transform the electromagnetic wave spectrum (dispersive, nonlinear, tunnel characteristics of photonic structures). Currently, the basic researches (more than $80 \%$ ) are concentrated on the twodimensional photonic crystals that have functionality of three-dimensional photonic crystals and rather simple manufacturing techniques and are directed towards the development of active and passive elements of the future integrated nanophotonic circuits (two-dimensional photonic crystal nanolasers with quantum wells and quantum dots; photonic waveguides, photonic fibres).

The perspective material for development of twodimensional photonic crystals is the macroporous silicon due to formation of structures of the necessary geometry, the high ratio of the cylindrical macropore depth to the diameter. Macroporous silicon structures are perspective for the development of thermal detectors, photonic waveguides due to occurrence of the powerful absorption bands in the infrared spectral range. Formation of the macroporous silicon structures with nanocoatings expands its functionalities as light emitters and photodetectors.

\section{Referencies}

1. E. Yablonovich, Inhibited spontaneous emission in solid-state physics and electronics // Phys. Rev. B42 (11), p. 846-851 (1987).

2. S. John, Strong localization of photonic in certain disordered dielectric structures // Phys. Rev. Lett. $\mathbf{5 8}$ (23), p. 2486-2489 (1987).

3. M. Augustin, R. Iliev, H.-J. Fuchs, et al., Highly efficient waveguide bends in low in-plane index contrast photonic crystals // Photonic Crystal 
Materials and Devices II, Proc. SPIE 5360, p. 156164 (2004).

4. T. Yoshie, M. Longar, K. Okamoto, etc., Photonic crystal nanocavities with quantum well or quantum dot active material // Ibid. 5360, p. 16-23 (2004).

5. L.A. Karachevtseva, O.A. Litvinenko, E.A. Malovichko, Stabilization of electrochemical formation of macropores in $\mathrm{n}$-Si // J. Theor. and Experim. Chem. 34 (5), p. 314-318 (1998).

6. A. Maradudin, A.R. McGurn, Out of plane propagation of electromagnetic waves in a twodimensional periodic dielectric medium // J. Mod. Opt. 41 (2), p. 275-284 (1994).

7. M.M. Sigalas, R. Bismas, K.M. Ho, and C.M. Soukoulis, Theoretical investigation off-plane propagation of electromagnetic waves in twodimensonal photonic crystals // Phys. Rev. B58 (11), p. 6791-6794 (1998).

8. L.A. Karachevtseva, O.A. Litvinenko, E.I. Stronska, Development and optical characteristics of the macroporous silicon structures // Semiconductor
Physics, Quantum Electronics and Optoelectronics, 3 (1), p. 22-25 (2000).

9. L.A. Karachevtseva, O.A. Litvinenko, E.A. Malovichko, E.I. Stronska, Optical transmittance of 2D macroporous silicon structures // Ibid. 4(4), p. 347-351 (2001).

10. R.Yu. Holiney, L.A. Matveeva, E.F. Venger, O.A. Litvinenko, and L.A. Karachevtseva, Electroreflectance study of macroporous silicon structures // Appl. Surf. Sci. 172 (3), p. 214-219 (2001).

11. L. Karachevtseva, M. Karas', V. Onishchenko, F. Sizov, Enhancement of the photoconductivity in 2D photonic macroporous silicon structures // Photonic Crystal Materials and Devices II, Proc. SPIE 5360, p. 381-389 (2004).

12. L.A. Karachevtseva, O.A. Lytvynenko, Y. Fukuda, and K. Furuya, Photoluminescence of complex microporous-macroporous silicon structures // Material Science and Material Properties for Infrared Optoelectronics, Proc. SPIE 4355, p. 146154 (2001). 